A - preparing concepts

$\mathrm{B}$ - formulating methods

$\mathrm{C}$ - conducting research

$\mathrm{D}$ - processing results

E - interpretation and conclusions

$\mathrm{F}$ - editing the final version

\section{Comparative effects of two exercise training programs on health-related quality of life in middle-aged women with non-alcoholic fatty liver disease}

\author{
Ahmad Mahdi Ahmad*1,A,B,D-F (iD, Heba Mohammed Ali $2, A-C, F$ iD \\ ${ }^{1}$ Department of Physical Therapy for Cardiovascular and Respiratory \\ Disorders, Faculty of Physical Therapy, Cairo University, Egypt \\ ${ }^{2}$ Department of Physical Therapy for Internal Medicine, Faculty of Physical \\ Therapy, Beni-Suef University, Egypt
}

*Correspondence: Ahmad Mahdi Ahmad; Faculty of Physical Therapy, Cairo University, Egypt; email: Ahmed.mahdy@pt.cu.edu.eg
Received: 2020-06-27

Accepted: 2020-09-27

Published: 2020-10-23

\title{
Abstract
}

Introduction: Insufficient research exists on the effectiveness of exercise therapy in non-alcoholic fatty liver disease (NAFLD), particularly regarding the health-related quality of life (HRQoL) outcome. Therefore, this study aimed to assess the effects of high-intensity interval training (HIT) compared to moderate-intensity combined resistance and aerobic training (RT/AT) on HRQoL in NAFLD patients.

Material and methods: Twenty-three middle-aged obese women with NAFLD were enrolled in 3-month exercise interventions of either HIIT $\left(\mathrm{n}_{1}=12,46.3 \pm 5\right.$ years, $\left.40.5 \pm 4.1 \mathrm{~kg} / \mathrm{m}^{2}\right)$ or moderate-intensity combined RT/AT $\left(\mathrm{n}_{2}=11\right.$, $45.6 \pm 4.4$ years, $43.5 \pm 5.4 \mathrm{~kg} / \mathrm{m}^{2}$ ). Secondary hepatic steatosis, liver cirrhosis, or virus C hepatitis were excluded. HIIT was prescribed as 4 sets $x 4$ min at $80-85 \%$ HRmax with 3 min active recovery. Combined RT/AT was prescribed as 10 min low to moderate cycling exercises followed by 8 resistance exercises at 50-75\% 1-RM. The outcomes were HRQoL, as assessed by the Chronic Liver Disease Questionnaire (CLDQ), and the percentage of body weight change ( $\% \Delta \mathrm{BW}$ ).

Results: CLDQ domains were significantly improved in both HIIT \& RT/AT groups compared to baselines; the emotional function \& worry domains and the total CLDQ score were significantly higher in the HIIT group ( $p<0.05$ ) compared to the other group. Body weight was reduced by $13 \% \& 8 \%$ in HIIT and combined RT/AT groups, respectively, with a significant difference in the $\% \Delta \mathrm{BW}$ between the two groups in favor of HIIT.

Conclusions: Both HIIT and moderate-intensity combined RT/AT could be suggested to improve the HRQoL and reduce body weight in middle-aged obese women with NAFLD, however, HIIT could be superior to the combined RT/AT in improving overall HRQoL, especially the emotional aspects, and reducing body weight.

Keywords: exercise, non-alcoholic fatty liver disease, quality of life, body weight change, high-intensity interval training

\section{Introduction}

Non-alcoholic fatty liver disease (NAFLD) has become a leading cause of chronic liver disease world- wide and has been estimated to affect $25 \%$ of people around the world with the highest prevalence being in the Middle East region (32\%) [1,2]. NAFLD can increase the risk for the end-stage hepatic disease in which

This is an Open Access journal, all articles are distributed under the terms of the Creative Commons Attribution-NonCommercial-ShareAlike 4.0 International (CC BY-NC-SA 4.0). License (http://creativecommons. org/licenses/by-nc-sa/4.0/). 
hepatocellular carcinoma (HCC) can develop [3]. Patients with NAFLD experience physical and psychological symptoms such as fatigue, physical inactivity, anxiety, depression, and reduced self-esteem that negatively impact patients' health-related quality of life (HRQoL) [4]. The HRQoL has been found to be significantly lower in women with NAFLD than in men counterparts, besides, a significantly negative correlation has been found between the low HRQoL and the presence of obesity [5]. The treatment plan of NAFLD should incorporate strategies to improve the physical and psychological aspects of HRQoL [6], particularly in obese women with NAFLD.

Current guidelines recommend lifestyle interventions, including diet and physical exercises, as major strategies for the treatment of almost all patients with NAFLD [7,8]. Exercise-based clinical trials have shown beneficial effects in patients with NAFLD concerning disease-related outcomes such as intrahepatic lipid content, liver enzymes, and markers of hepatocellular injury [9]. However, limited research work has been done on the exercise-induced changes in the HRQoL, independent of caloric restriction, in patients with NAFLD. Only one study has been found in this regard and showed that the high-intensity interval training (HIIT) led to significant improvements in HRQoL in diabetic patients with NAFLD [10]. Because of this, more research work is needed to confirm the exercise-induced benefits in the HRQoL outcome in isolated NAFLD and to clarify the exercise training type that yields better benefits in HRQoL in patients with NAFLD. Therefore, in an attempt to fill this research gap, this study aimed to assess and compare the effects of the HIIT and the moderate-intensity combined resistance \& aerobic training (RT/AT), as two commonly used interventions in exercise training settings, on the HRQoL and the percentage of body weight change in middle-aged obese women with NAFLD. The results of this research may aid current efforts directed to reduce the overall burden of NAFLD on patients' quality of life and direct future exercise-based interventions in this regard.

\section{Materials and methods}

Twenty-three patients with non-alcoholic fatty liver disease (NAFLD), recruited by physician referral, have completed this study. The diagnosis of NAFLD was confirmed by abdominal ultrasonography, as the firstline imaging method used in clinical settings in suspected NAFLD [11], with evidence of hepatic steatosis and absence of secondary causes of hepatic steatosis as significant alcohol intake, chronic use of steatogenic medications, and/or hereditary disorders [7]. The inclusion and exclusion criteria were evaluated and confirmed by the physician. The inclusion criteria were NAFLD, women patients with simple hepatic steatosis, middle-aged adults (ages 36-55 years), patients with class II and III obesity (BMI of $\geq 35 \mathrm{~kg} / \mathrm{m}^{2}$ ), patients with blood pressure $<140 / 90$, and patients with glycosylated hemoglobin $<6.5 \%$. The exclusion criteria were patients with secondary causes of liver steatosis, patients with liver fibrosis or cirrhosis and/or end-stage liver disease, patients with hepatitis B or hepatitis $\mathrm{C}$ infection, patients with hypertension or diabetes, pregnant, patients with cardiovascular or pulmonary disorders, patients with musculoskeletal or neurological limitations to exercise training, and/or patients participating in other exercise programs. Eligible patients were allocated by consecutive sampling into two groups; High-intensity interval training (HIIT) group $\left(\mathrm{n}_{1}=12\right)$ and combined resistance $\&$ aerobic training (RT/AT) group $\left(\mathrm{n}_{2}=11\right)$. The flow of patients throughout the study can be found in the flowchart shown in Figure 1. Patients in both groups were instructed to maintain the dietary advice given to them by their physician throughout the intervention.

\section{Anthropometric evaluation}

At the baseline, body weight and height were measured using calibrated weight and height scale equipment, and the body mass index (BMI) for each patient was calculated as BMI $\left(\mathrm{kg} / \mathrm{m}^{2}\right)=$ body weight $(\mathrm{kg}) / \mathrm{he}-$ ight $\left(\mathrm{m}^{2}\right)$. After the interventions, the body weight was measured once again.

\section{HRQoL evaluation by Chronic Liver Disease Questionnaire (CLDQ)}

The HRQoL was evaluated as a primary outcome of the study at the baseline and post-intervention utilizing the Chronic Liver Disease Questionnaire (CLDQ). The CLDQ is a fully validated questionnaire that can be used to assess the HRQoL for all types of liver diseases including NAFLD and has been developed by Younossi et al. [12]. CLDQ includes 29 items in the following domains: abdominal symptoms domain (3 items), fatigue domain (5 items), systemic symptoms domain (5 items), activity domain (3 items), emotional function domain (8 items), and worry domain (5 items) [12]. The answers to each item were Likert scale type of answers, ranging from 1 "all of the time" or (the most impairment) to 7 "none of the time" or (the least impairment). Scoring of the CLDQ domains was done in our study according to Younossi et al. [12]. The score of each domain of the CLDQ was calculated by adding the sub-scores of the specific items included in this particular domain and then dividing by the number of these items [12]. The overall CLDQ score was determined by adding the sub-scores of all items included in 


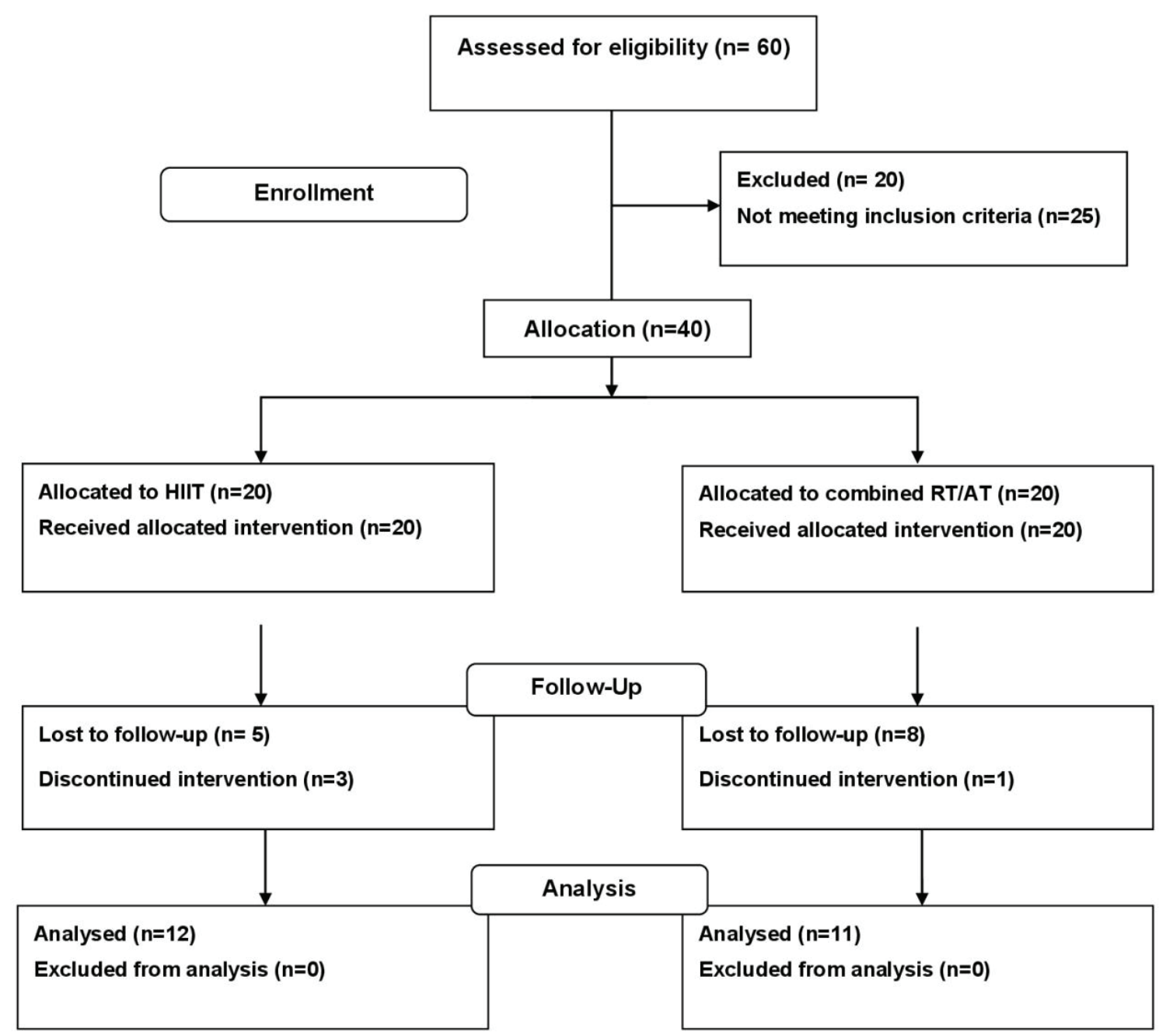

Fig. 1. The flowchart of the study

the questionnaire and then dividing by the total number of items $(n=29)$, with a higher overall score indicating a better health-related quality of life $[12,13]$.

\section{Percentage of body weight change $(\% \Delta B W)$}

The $\% \Delta \mathrm{BW}$ was calculated for each patient after completion of the interventions as: $\Delta \mathrm{BW}(\%)=($ the post-intervention $\mathrm{BW})$ - (the pre-intervention $\mathrm{BW}) /($ the pre-intervention $\mathrm{BW}) \times 100$.

\section{Determination of one-repetition maximum (1-RM )}

The maximum load that can be lifted for one time through a full range of motion or the one-repetition maximum (1-RM), necessary for the determination of the intensity of resistance exercises, was determined according to Fleck and Kraemer [14]. The muscle groups tested for the 1-RM were those involved in biceps curl, triceps press, chest press, shoulder press, lateral pull-down, leg extension, seated hamstrings curl, and calf raises. The patients were instructed to gradually increase the load they lift until they achieved the maximum load that only could be lifted for one time through the full range of motion (1-RM load). Rest periods were permitted in-between the attempts during the test to reduce muscle fatigue which could underestimate the 1-RM load.

\section{Predicted maximum heart rate calculation}

The values of the maximal heart rate $\left(\mathrm{HR}_{\max }\right)$ of patients were predicted according to this equation: predicted $\mathrm{HR}_{\max }=220$-age [15]. The predicted $\mathrm{HR}_{\max }$ was necessary for the calculation of the target heart rate during HIIT.

\section{Dietary instructions}

All patients received dietary instructions focusing on a healthy balanced diet without caloric restriction prior to exercise training. Information provided included a description of the healthy food composition rather than a calculation of the number of calories consumed per day. 


\section{Exercise training interventions}

All exercise training sessions in both groups were supervised by the physiotherapist and were scheduled for three alternating days per week for 12 weeks.

\section{High-intensity interval training (HIIT)}

The training sessions were conducted in the Fitness \& Rehabilitation Unit at kasr Alainy Hospital, Cairo, Egypt. The HIIT was in the form of 4 sets $\times$ $4 \mathrm{~min}$ of high-intensity exercises separated by $3 \mathrm{~min}$ active recovery period, as reported by Hamasaki [16]. At first, a 5-10 min warm-up period of mild walking was allowed. Then, the conditioning phase of exercise protocol was prescribed as 4 intervals $\times 4$ min of highintensity training (HIIT) on a treadmill at $80-85 \%$ of predicted $\mathrm{HR}_{\max }$ with 3 min active recovery interval in between at $65-75 \%$ of predicted $\mathrm{HR}_{\max }$. Finally, a 5 min cool down period was permitted. In the beginning, during the first two weeks, the patients were allowed not to complete the whole bouts of the HIIT upon their request due to their initially lower cardiorespiratory fitness. However, by the third week, all patients were able to complete the entire HIIT session. All patients were exercising using a heart rate monitoring device cleared by the FDA (Datex-Ohmeda 3900 Pulse Oximeter, Made in USA) to ensure that each patient was exercising within the individualized target heart rate. The total duration of the HIIT session was 35-40 minutes.

\section{Moderate-intensity combined resistance and aerobic training (RT\&AT)}

The exercise session began with 10 minutes of aerobic cycling exercises at low to moderate intensity. Then, patients performed 8 different resistance exercises involving major muscle groups using free weights; the muscle groups were biceps curl, triceps press, chest press, shoulder press, lateral pull-down, leg extension, seated hamstrings curl, and calf raises. The number of repetitions was 8-12 repetitions per set, for 2-3 sets of each exercise, and with 2 min rest in between sets [17]. The resistance exercises were performed at a moderate intensity of $50-75 \% 1-\mathrm{RM}$. In the first two weeks, the exercises were carried out at an intensity of $50 \%$ of 1-RM, then, the load was gradually increased and all patients exercised at $75 \%$ of 1-RM since the beginning of the $5^{\text {th }}$ week till the end of the intervention. At the end of the exercise session, 5 min cooling down was permitted using low-intensity cycling exercises. The entire duration of the combined RT/AT session was $45-50$ minutes. The timeline of the study is presented in Figure 2.

\section{Statistical analysis}

Shapiro-Wilk test was used to assess the normality of data distribution. Descriptive statistics were used to describe the data as means \pm standard deviations. Inferential statistics were used to analyze the data; the one-way ANOVA test was used to assess the difference between the two groups at the baseline and after the interventions. One-way repeated measures ANOVA test was used to assess the pre- and post-intervention changes within each group. The ANCOVA test was used to compare fatigue and systemic symptoms domains postintervention after adjusting for the baseline differences in these domains between the two groups. Data were presented as means \pm standard deviations and $p$ values of $<0.05$ were considered as statistically significant. Social science statistics and VassarStats software programs were used for statistical analysis.

\section{Ethical consideration}

The study protocol has been approved by the Ethics Committee of Scientific Research of the Faculty of

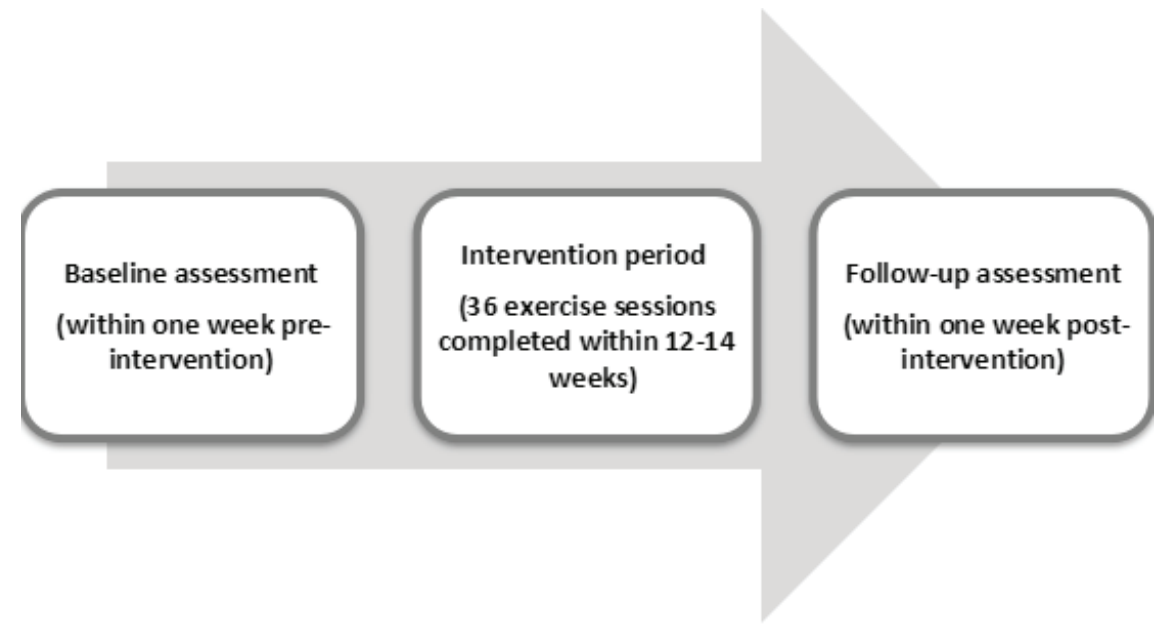

Fig. 2. The timeline of the study 
Tab.1. Baseline patients' characteristics

\begin{tabular}{|c|c|c|c|c|}
\hline \multicolumn{2}{|c|}{ Variables } & HIIT group $\left(\mathrm{n}_{1}=12\right)$ & Combined RT/AT group $\left(\mathrm{n}_{2}=11\right)$ & p-value \\
\hline \multicolumn{2}{|c|}{ Age (years) } & $46.30 \pm 5.00$ & $45.60 \pm 4.40$ & 0.693 \\
\hline \multicolumn{2}{|c|}{ Body weight (kg) } & $106.00 \pm 13.90$ & $108.00 \pm 14.00$ & 0.735 \\
\hline \multicolumn{2}{|c|}{ BMI $\left(\mathrm{kg} / \mathrm{m}^{2}\right)$} & $40.50 \pm 4.10$ & $43.50 \pm 5.40$ & 0.145 \\
\hline \multirow{7}{*}{ CLDQ } & Abdominal symptoms & $3.36 \pm 0.52$ & $3.39 \pm 0.33$ & 0.858 \\
\hline & Fatigue & $2.78 \pm 0.87$ & $2.22 \pm 0.33$ & 0.055 \\
\hline & Systemic symptoms & $3.17 \pm 0.69$ & $2.60 \pm 0.40$ & $0.027 *$ \\
\hline & Activity & $3.56 \pm 0.85$ & $4.24 \pm 0.84$ & 0.144 \\
\hline & Emotional function & $3.11 \pm 0.88$ & $3.16 \pm 0.43$ & 0.881 \\
\hline & Worry & $3.00 \pm 0.93$ & $2.67 \pm 0.57$ & 0.327 \\
\hline & Total score & $3.09 \pm 0.51$ & $2.96 \pm 0.31$ & 0.491 \\
\hline
\end{tabular}

Data are expressed as means \pm standard deviations; BMI, body mass index; CLDQ, Chronic Liver Disease Questionnaire; * significant $p$-value $(<0.05)$ from the one-way ANOVA test.

Physical Therapy at Cairo University and has followed the tenets of the Declaration of Helsinki. The patients provided their written consent before participating in the study.

\section{Results}

At the baseline, there was a non-significant difference in anthropometric measures between the two groups, as shown in Table 1. However, there was a quite non-significant difference in the fatigue domain of the CLDQ $(p=0.055)$ and a significant difference in the systemic symptoms domain of the CLDQ $(p=0.027)$ between the two groups, as shown in Table 1. At the end of the interventions, there were significant differences in the scores of all CLDQ domains compared to the baseline values in both exercise groups $(p<0.05)$. Also, the CLDQ emotional function domain $(\mathrm{p}=0.010)$, the CLDQ worry domain ( $p=0.047)$, as well as the total CLDQ score $(p=0.023)$ were significantly higher in the HIIT group compared to the moderate-intensity combined RT/ AT group at the end of the interventions, as shown in Table 2 . The body weight has reduced by about $13 \%$ in the HIIT group and $8 \%$ in the moderate-intensity combined RT/AT group, with a significant difference in the percentage of body weight change $(\% \Delta B W)$ between the two exercise groups in favor of the HIIT group ( $\mathrm{p}=0.036)$, as shown in Table 2 .

\section{Discussion}

The HRQoL has been considered as an essential outcome in the interventional research work. Patients with NAFLD have debilitated HRQoL, nevertheless, they receive little attention in this regard than other patient populations. Thus, we have conducted this exercise-based intervention to shed light on HRQoL changes in response to two different forms of exercise training in NAFLD patients. The impact of exercise training on HRQoL, without concurrent dietary caloric restriction, has been studied less extensively in patients with chronic liver diseases. We found one study in patients with liver cirrhosis [18], another study in patients waiting for liver transplantation [19], and one more study in patients after liver transplantation [20]. Likewise, there is limited data regarding the independent effect of exercise training on HRQoL in patients with NAFLD, with only one study found in this regard [10]. Up to our knowledge, our study is the first one to compare the effect of HIIT with the effect of moderate-intensity combined RT/AT on HRQoL in NAFLD patients. The major findings in this study are: (a) Both HIIT and moderate-intensity combined RT/AT have induced significant improvements in all domains of CLDQ compared to the baseline in middle-aged obese women with NAFLD. (b) HIIT was superior to the moderate-intensity combined RT/AT in improving the emotional aspects of HRQoL (i.e. emotional function and worry domains of CLDQ) and the overall score of CLDQ in these patients. 
Tab. 2. Results of the exercise groups after the interventions

\begin{tabular}{llccccc}
\hline \multirow{2}{*}{ Variables } & \multicolumn{3}{c}{ HIIT group $\left(\mathrm{n}_{1}=12\right)$} & \multicolumn{2}{c}{$\begin{array}{c}\text { Combined RT/AT group } \\
\left(\mathrm{n}_{2}=11\right)\end{array}$} & $\begin{array}{c}\text { HIIT versus RT/AT } \\
\text { after interventions }\end{array}$ \\
\hline & & Pre & Post & Pre & Post & p-value \\
\hline CLDQ & Abdominal symptoms & $3.36 \pm 0.52$ & $6.42 \pm 0.32^{*}$ & $3.39 \pm 0.33$ & $6.15 \pm 0.52^{*}$ & 0.153 \\
& Fatigue & $2.78 \pm 0.87$ & $5.98 \pm 0.34^{*}$ & $2.22 \pm 0.33$ & $5.69 \pm 0.33^{*}$ & 0.379 \\
& Systemic symptoms & $3.17 \pm 0.69$ & $5.93 \pm 0.21^{*}$ & $2.60 \pm 0.40$ & $5.69 \pm 0.34^{*}$ & 0.249 \\
& Activity & $3.56 \pm 0.85$ & $6.61 \pm 0.37^{*}$ & $4.24 \pm 0.84$ & $6.45 \pm 0.31^{*}$ & 0.293 \\
& Emotional function & $3.11 \pm 0.88$ & $6.16 \pm 0.45^{*}$ & $3.16 \pm 0.43$ & $5.67 \pm 0.36^{*}$ & $0.010^{\S}$ \\
& Worry & $3.00 \pm 0.93$ & $6.00 \pm 0.47^{*}$ & $2.67 \pm 0.57$ & $5.67 \pm 0.22^{*}$ & $0.047^{\S}$ \\
& Total score & $3.09 \pm 0.51$ & $6.08 \pm 0.27^{*}$ & $2.96 \pm 0.31$ & $5.80 \pm 0.27^{*}$ & $0.023^{\S}$ \\
The $\Delta \mathrm{BW}(\%)$ & $-12.92 \pm 5.75$ & & $-7.92 \pm 4.86$ & $0.036^{\S}$ \\
\hline
\end{tabular}

Data are expressed as means \pm standard deviations; CLDQ, Chronic Liver Disease Questionnaire; $\Delta$ BW, body weight change; * significant change from the repeated measures ANOVA test; ${ }^{\S}$ significant $p$-value $(<0.05)$ from the one-way ANOVA test.

(c) the HIIT and the moderate-intensity combined RT/ AT induced clinically significant reductions in the BW by $13 \% \& 8 \%$, respectively, in obese middle-aged women with NAFLD compared to the baseline. These percentages of body weight change were significantly different for the sake of the HIIT.

In our study, significant improvements in all CLDQ domains were reported in the HIIT group compared to the baseline. This finding was in accordance with Abdelbaset et al. [10], who have recently shown that 8-week HIIT exercise training led to significant improvements in CLDQ scores compared to the baseline in diabetic patients with NAFLD. Likewise, the HRQoL domains were improved significantly in the moderateintensity combined RT/AT group compared to the baseline. This finding could be supported by Moya-Nájera et al. [20] who showed that 24-weeks combined aerobic and resistance training produced significant improvement in the HRQoL, assessed by SF-12 Health Survey Questionnaire, compared to the baseline in patients received liver transplantation. Also, a combined exercise training comprising flexibility, resistance, and aerobic exercises has been associated with a better HRQoL than the non-exercise group [21]. The mechanism underlying the significant improvement in the overall HRQoL in our exercise groups, as evidenced by the significant changes in the CLDQ total score compared to the baseline, could be the loss of body weight observed in the two groups. This explanation can be supported by Tapper and Lai [22], who have found that weight loss led to significant improvement in the HRQoL in patients with NAFLD as measured by CLDQ. They implied that this improvement was specific to the loss of body weight and not to the biochemical improvements, simply because NAFLD patients who lose weight can better increase their daily activity commitments [22].

With respect to fatigue and activity domains of CLDQ, the significant improvements in these domains compared to the baseline in our exercise groups could be attributed to a possible increase in cardiorespiratory fitness and muscle strength from exercise training. This explanation can be supported by reports indicating that participation in exercise training generally tends to be associated with physical benefits such as improved fitness levels [23]. With regard to the emotional function and worry domains of CLDQ, the significant improvements in these domains compared to the baseline in our exercise groups could be explained by the exercise-induced sensations of improved wellbeing and mood, a decline in anxiety and depression symptoms, and even euphoric feelings [24]. There are psychological and physiologic hypotheses underlying these effects. Psychological hypotheses involve distraction, enhanced self-efficacy, and/or social interaction mechanisms [25]. The distraction mechanism suggests that physical exercises lead to a distraction from unfavorable stimuli, worries, and depressing thoughts [25]. The enhanced self-efficacy mechanism proposes that exercise training practice can be perceived as a challenging mission which when accomplished properly leads to improvement in self-confidence and a decline in negative self-evaluation [25]. The social interaction 
hypothesis suggests that social relationships and mutual support that occur between exercise participants play an important role in the effects of exercise on social well-being [25]. The physiological hypotheses include thermogenic, endorphin, and/or monoamine mechanisms $[25,26]$. The thermogenic hypothesis suggests that the rise in core body temperature following exercise would lead to an increase in the temperature of the brain stem resulting in an overall feeling of relaxation and reduction in symptoms of anxiety [26]. The endorphin mechanism suggests that an increased release of the $\beta$-endorphins following exercise is related to a positive mood and overall enhanced sensations of well-being [26]. The monoamine mechanism suggests that exercise leads to an increase in the bioavailability of brain neurotransmitters (e.g. serotonin, dopamine, and norepinephrine) which may contribute to the antidepressant and anxiolytic effects of exercise [26]. It should be pointed out that heavy or high-intensity exercises near maximal work capacity, as performed by elite athletes, are associated with a worsened mood [25]. Obviously, this does not apply to the HIIT in our study, in which the highintensity bouts were performed at a submaximal heart rate (i.e. at $80-85 \%$ of predicted $\mathrm{HR}_{\max }$ ) and the active recovery bouts were performed at $65-75 \%$ of predicted $\mathrm{HR}_{\max }$.

Upon comparison between the two exercise training groups in our study, the emotional function and the worry domains were the only CLDQ domains that were significantly higher in the HIIT group compared to the moderate-intensity combined RT/AT group after the interventions. This finding may be supported by other studies; Choi et al. [27], showed that post-exercise psychological states and depression items of the Hospital Anxiety and Depression Scale were significantly improved in the HIIT group compared to the moderateintensity continuous training group in post-myocardial infarction patients, in addition to, a notably greater reduction in insomnia in the HIIT group compared to the other group. Another study by Abdelhalem et al. [28], showed that the high-intensity exercise group showed better improvement in the role emotional domain of the Short Form 36-Item (SF-36) Health Survey than the moderate-intensity exercise group in patients with coronary artery disease. In these two studies [27,28], although the sample population and the comparative exercise protocols were not the same as in our study, both studies reported that exercise training of higher intensity has offered more health benefits in the psychological and/or emotional states of the participants than the moderate-intensity exercise type. Moreover, a recent meta-analysis by Oliveira et al.[29] showed that HIIT training with adequate recovery intervals can produce greater improvement in affective psychological responses to exercise in healthy normal-weight and obese individuals. On the other hand, a combined resistance and aerobic training failed to show a significant improvement in the role emotional domain of the SF-36 Health Survey compared to the controls in liver transplant patients [20]. Another important finding in our study regarding the HRQoL was the significantly higher total score of CLDQ in the HIIT group compared to the moderate-intensity combined RT/AT group after the interventions. Evidence indicates that physical activity/ exercises of higher intensities are associated with better overall HRQoL [30-33]. The physiological mechanisms underlying the superiority of the HIIT for the overall HRQOL could be attributed to higher recruitment of muscle fibers and greater gains in maximum oxygen consumption $\left(\mathrm{Vo}_{2} \mathrm{max}\right)$ which would result in increased fitness and better physical health [34]. Besides, it has been shown that enjoyment responses were higher in the HIIT compared to either moderate-intensity or vigorous-intensity continuous training [34,35], which could result in better mood and emotional function.

The last findings in our study include the clinically significant reductions in the body weight observed in the two exercise groups compared to the baseline and the superiority of HIIT over the combined RT/AT regarding the percentage of body weight reduction. The BW was reduced by about $13 \%$ \& $8 \%$ in the HIIT and the moderate-intensity combined RT/AT groups, respectively, from the baseline. Notably, these weight reductions came in accordance with the current guidelines of the management of NAFLD which have set a target weight loss of 7\%-10\% from the baseline as a recommendation for lifestyle interventions such as exercise interventions $[7,8]$. This finding may be supported by Banitalebi et al. [36], who have reported significant changes in body weight compared to the baseline following 10 weeks of both HIIT and combined aerobic/ resistive training in type 2 diabetic women. Also, Willis et al. [37], have shown that aerobic training and combined aerobic/ resistance training have reduced body weight significantly in obese adults compared to the baseline. Furthermore, Khammassi et al. [38], have shown that a 12-week HIIT program, without dietary intervention, has induced a significant change in body weight compared to the baseline in healthy obese subjects. The clinical relevance of weight loss in NAFLD patients has been reported by earlier studies. It has been shown that a weight loss of $\geq 7 \%$ improves liver histology and cardio-metabolic profile in patients with NAFLD [39]. Weight loss also reduces liver biochemical tests, intrahepatic fat content, serum lipids, and insulin in NAFLD [40,41]. Lastly, it is important to mention that no clinically adverse events were identified over the course of the study in either group. 
As with the majority of studies, our findings have to be seen in light of some limitations. The main limitations to the generalization of our results are the relatively small sample size of the study resulted from the increased dropouts and the non-randomized allocation of the patients. Also, relying on a regression equation to estimate the age-predicted maximum heart rate $\left(\mathrm{HR}_{\max }\right)$ instead of direct $\mathrm{HR}_{\text {max }}$ measurement by a graded exercise test represents another limitation. Furthermore, adherence statistics and patients' compliance to the dietary instructions were not assessed in this study. Nevertheless, our study has a number of strengths, which include being the first study to assess and compare the different effects of two common types of exercise training on the HRQoL in patients having isolated NAFLD without coexisting diabetes or cardiovascular diseases, in whom the baseline HRQoL was only affected by the health issues related to the NAFLD itself and not to a combination with other co-existing medical conditions. Besides, the exercise training interventions implemented in our study were closely supervised by the physiotherapist.

\section{Conclusions}

A definite conclusion cannot be drawn because of the small sample size of the study, nevertheless, we can suggest that both HIIT and moderate-intensity combined RT/AT may be effective exercise-based interventions to improve the health-related quality of life and induce clinically significant reductions in body weight in obese middle-aged women with NAFLD. In addition, the HIIT may be proposed as a superior intervention to the moderate-intensity combined RT/AT for improving the overall HRQoL, particularly, the emotional healthrelated quality of life, and reducing body weight in this patient population. Finally, our findings can be viewed as preliminary observations that may guide future research work in the area of exercise/physical activity promotion for better HRQoL of patients with NAFLD.

\section{Funding}

This research received no external funding.

\section{Conflicts of interest}

The authors declare no conflict of interest.

\section{References}

1. Younossi ZM, Koenig AB, Abdelatif D, Fazel Y, Henry L, Wymer M. Global epidemiology of nonalcoholic fatty liver disease-meta-analytic assessment of pre- valence, incidence, and outcomes. Hepatology. 2016 July; 64(1): 73-84.

2. Mundi MS, Velapati S, Patel J, Kellogg TA, Abu Dayyeh BK, Hurt RT. Evolution of NAFLD and Its Management. Nutr Clin Pract. 2020 Feb; 35(1): 72-84.

3. Lindenmeyer CC, McCullough AJ. The Natural History of Nonalcoholic Fatty Liver Disease-An Evolving View. Clin Liver Dis. 2018 Feb; 22(1): 11-21.

4. Golabi P, Otgonsuren M, Cable R. et al. Non-alcoholic Fatty Liver Disease (NAFLD) is associated with impairment of Health Related Quality of Life (HRQOL). Health Qual Life Outcomes. 2016 Feb 9; 14: 18.

5. Huber Y, Boyle M, Hallsworth K, et al. Health-related Quality of Life in Nonalcoholic Fatty Liver Disease Associates With Hepatic Inflammation. Clin Gastroenterol Hepatol. 2019 Sep; 17(10): 2085-2092.e1.

6. Assimakopoulos K, Karaivazoglou K, Tsermpini EE, Diamantopoulou G, Triantos C. Quality of life in patients with nonalcoholic fatty liver disease: A systematic review. J Psychosom Res. 2018 Sep; 112: 73-80.

7. Chalasani N, Younossi Z, Lavine JE, et al. The diagnosis and management of nonalcoholic fatty liver disease: Practice guidance from the American Association for the Study of Liver Diseases. Hepatology. 2018 Jan; 67(1): 328-57.

8. Leoni S, Tovoli F, Napoli L, Serio I, Ferri S, Bolondi L. Current guidelines for the management of nonalcoholic fatty liver disease: A systematic review with comparative analysis. World J Gastroenterol. 2018 Aug 14; 24(30): 3361-73.

9. Wang S, Zheng J, Peng H, et al. Physical activity intervention for non-diabetic patients with non-alcoholic fatty liver disease: a meta-analysis of randomized controlled trials. BMC Gastroenterol. 2020 March 12; 20: 66 .

10. Abdelbasset WK, Tantawy SA, Kamel DM, Alqahtani BA, Soliman GS. A randomized controlled trial on the effectiveness of 8-week high-intensity interval exercise on intrahepatic triglycerides, visceral lipids, and health-related quality of life in diabetic obese patients with nonalcoholic fatty liver disease. Medicine (Baltimore). 2019 Mar; 98(12): e14918.

11. Zhou JH, Cai JJ, She ZG, Li HL.Noninvasive evaluation of nonalcoholic fatty liver disease: Current evidence and practice. World J Gastroenterol. 2019 Mar 21; 25(11): 1307-26.

12. Younossi ZM, Guyatt G, Kiwi M, Boparai N, King D. Development of a disease specific questionnaire to measure health related quality of life in patients with chronic liver disease. Gut. 1999 Aug; 45(2): 295-300.

13. Chawla KS, Talwalkar JA, Keach JC, Malinchoc M, Lindor KD, Jorgensen R. Reliability and validity of the Chronic Liver Disease Questionnaire (CLDQ) in 
adults with non-alcoholic steatohepatitis (NASH). BMJ Open Gastroenterol. 2016 Mar 16; 3(1): e000069.

14. Fleck SJ, Kraemer W. Designing resistance training programs. 4th ed. Champaign, IL: Human Kinetics; 2014.

15. Gibson AL, Wagner DR, Heyward VH. Advanced Fitness Assessment and Exercise Prescription. 8th ed. Champaign, IL: Human Kinetics; 2019.

16. Hamasaki H. Perspectives on Interval Exercise Interventions for Non-Alcoholic Fatty Liver Disease. Medicines (Basel). 2019; 6(3): 83.

17. Zelber-Sagi S, Buch A, Yeshua H, et al. Effect of resistance training on non-alcoholic fatty-liver disease a randomized-clinical trial. World J Gastroenterol. 2014 Apr $21 ; 20(15): 4382-92$.

18. Román E, Torrades MT, Nadal MJ, et al. Randomized pilot study: effects of an exercise programme and leucine supplementation in patients with cirrhosis. Dig Dis Sci. 2014 Aug; 59(8):1966-75.

19. Debette-Gratien M, Tabouret T, Antonini MT, et al. Personalized adapted physical activity before liver transplantation: acceptability and results. Transplantation 2015 Jan; 99(1): 145-50.

20. Moya-Nájera D, Moya-Herraiz Á, Compte-Torrero L, et al. Combined resistance and endurance training at a moderate-to high intensity improves physical condition and quality of life in liver transplant patients. Liver Transpl 2017 Oct; 23(10): 1273-81.

21. Choi MJ, Park YG, Kim YH, Cho KH, Nam GE. Association between type of exercise and health-related quality of life in adults without activity limitations: a nationwide cross-sectional study. BMC Public Health. 2020 May 1; 20(1): 599.

22. Tapper EB, Lai M. Weight loss results in significant improvements in quality of life for patients with nonalcoholic fatty liver disease: A prospective cohort study. Hepatology. 2016; 63(4): 1184-9.

23. Penedo FJ, Dahn JR. Exercise and well-being: a review of mental and physical health benefits associated with physical activity. Curr Opin Psychiatry. 2005 Mar; 18(2): 189-93.

24. Craft LL, Perna FM. The Benefits of Exercise for the Clinically Depressed. Prim Care Companion J Clin Psychiatry 2004; 6(3): 104-11.

25. Peluso MA, Guerra de Andrade LH. Physical activity and mental health: the association between exercise and mood. Clinics. 2005 Feb; 60(1): 61-70.

26. Landers DM, Arent SM. Physical activity and mental health. In: Tenenbaum G, Eklund RC, editors. Handbook of Sport Psychology. USA:Wiley, Hoboken, NJ; 2007. p. 469-90.

27. Choi HY, Han HJ, Choi JW, Jung HY, Joa KL. Superior Effects of High-Intensity Interval Training Compared to Conventional Therapy on Cardiovascular and
Psychological Aspects in Myocardial Infarction. Ann Rehabil Med. 2018 Feb; 42(1): 145-53.

28. Abdelhalem AM, Shabana AM, Onsy AM, Gaafar AE. High intensity interval training exercise as a novel protocol for cardiac rehabilitation program in ischemic Egyptian patients with mild left ventricular dysfunction. Egypt Heart J. 2018 Dec; 70(4): 287-94.

29. Oliveira BRR, Santos TM, Kilpatrick M, Pires FO, Deslandes AC. Affective and enjoyment responses in high intensity interval training and continuous training: a systematic review and meta-analysis. PLoS ONE. 2018 Jun 6; 13(6): e0197124.

30. Vuillemin A, Boini S, Bertrais S, et al. Leisure time physical activity and health-related quality of life. Prev Med. 2005; 41(2): 562-69.

31. Anokye NK, Trueman P, Green C, Pavey TG, Taylor RS. Physical activity and health related quality of life. BMC Public Health. 2012 Aug 7; 12: 624.

32. Cho KO. The Positive Effect of Physical Activity on Health and Health-related Quality of Life in Elderly Korean People-Evidence from the Fifth Korea National Health and Nutrition Examination Survey. J Lifestyle Med. 2014; 4(2): 86-94.

33. Kim S, Kwon YM, Park YI. Association between physical activity and health-related quality of life in Korean: the Korea National Health and nutrition examination survey IV. Korean J Fam Med. 2014; 35(3): 152-9. doi: 10.4082/kjfm.2014.35.3.152.

34. Kilpatrick MW, Jung ME, Little JP. High-intensity interval training: A review of physiological and psychological responses. ACSM s Health \& Fitness Journal 2014; 18(5): 11-6.

35. Bartlett JD, Close GL, MacLaren DPM, Gregson W, Durst B, Morton JP. High-intensity interval running is perceived to be more enjoyable than moderate-intensity continuous exercise: implications for exercise adherence. J Sport Sci. 2011; 29: 547Y53.

36. Banitalebi E, Faramarzi M, Nasiri S. High-Intensity Interval Training Versus Moderate Intensity Combined Training (Resistance and Aerobic) for Improving Insulin-Related Adipokines in Type 2 Diabetic Women. Zahedan J Res Med Sci. 2018 Nov 17; 20(10): e68793.

37. Willis LH, Slentz CA, Bateman LA, et al. Effects of aerobic and/or resistance training on body mass and fat mass in overweight or obese adults. J Appl Physiol. (1985). https://pubmed.ncbi.nlm.nih.gov/23019316/2012 Dec 15; 113(12): 1831-7.

38. Khammassi M, Ouerghi N, Hadj-Taieb S, Feki M, Thivel D, Bouassida A. Impact of a 12-week high-intensity interval training without caloric restriction on body composition and lipid profile in sedentary healthy overweight/obese youth. J Exerc Rehabil. 2018 Feb 26; 14(1): 118-25. 
39. Musso G, Cassader M, Rosina F, Gambino R .Impact of current treatments on liver disease, glucose metabolism and cardiovascular risk in non-alcoholic fatty liver disease (NAFLD): a systematic review and metaanalysis of randomised trials. Diabetologia. 2012 Apr; 55(4): 885-904.

40. Thoma C, Day CP, Trenell MI. Lifestyle interventions for the treatment of non-alcoholic fatty liver disease in adults: a systematic review. J Hepatol. 2012 Jan 1; 56(1): 255-66.

41. Brouwers B, Schrauwen-Hinderling VB, Jelenik T, et al. Exercise training reduces intrahepatic lipid content in people with and people without nonalcoholic fatty liver. Am J Physiol Endocrinol Metab. 2018 Feb7; 314(2): E165-E173. 